\title{
Cosmic-ray heating of molecular cloud cores
}

\author{
Daniele Galli* \\ INAF-Osservatorio Astrofisico di Arcetri, Largo E. Fermi 5, 50125 Firenze, Italy \\ E-mail: gallidarcetri.astro.it \\ Marco Padovani ${ }^{a, b}$ \\ ${ }^{a}$ Laboratoire Univers et Particules de Montpellier, UMR 5299 du CNRS, Université de \\ Montpellier II, place E. Bataillon, cc072, 34095 Montpellier, France \\ ${ }^{b}$ INAF-Osservatorio Astrofisico di Arcetri, Largo E. Fermi 5, 50125, Firenze, Italy \\ E-mail: Marco.PadovanidLupm.univ-montp2.fr
}

Cosmic rays are an important source of heating in the interstellar medium, in particular in dense molecular cloud cores shielded from the external ultraviolet radiation field. The limits placed on the cosmic-ray ionization rate from measurements of the gas temperature in dense clouds are unaffected by the uncertainties associated to the traditional methods based on the analysis of molecular abundances. However, high-resolution data are required to determine with sufficient accuracy the spatial temperature distribution within prestellar cores. In this contribution we illustrate in detail the case of the well-studied prestellar core L1544, showing that both its thermal structure and chemical composition are consistent with a cosmic ray ionization rate of $\sim 10^{-17} \mathrm{~s}^{-1}$, significantly smaller than the value measured in the diffuse interstellar medium. We also briefly discuss possible applications of this method to the molecular clouds of other galaxies.

Cosmic Rays and the InterStellar Medium

24-27 June 2014

Montpellier, France

\footnotetext{
* Speaker.
} 


\section{Introduction}

The recent discovery of significant amounts of $\mathrm{H}_{3}^{+}$in diffuse molecular clouds (e.g., Indriolo et al. 2007, 2009 and references therein) has spurred a significant increase of interest in the study of the propagation and ionization rate of low-energy cosmic rays (hereafter CRs) in the interstellar medium. A straightforward analysis of the observations of Indriolo et al. (2007) extended by Indriolo \& McCall (2012) to a sample of 50 diffuse molecular clouds, yields values of the ionization rate $\zeta$ for $\mathrm{H}_{2}$ in the range $\sim(2-11) \times 10^{-16} \mathrm{~s}^{-1}$, significantly larger than the "standard" value $\zeta \approx 10^{-17} \mathrm{~s}^{-1}$ that had been in use since the pioneering works of Hayakawa et al. (1961) and Spitzer \& Tomasko (1968). This unexpected result has led to the reconsideration of how CR protons and electrons propagate in the interstellar medium (e.g., Indriolo et al. 2009, Padovani et al. 2009, Padovani \& Galli 2011, Everett \& Zweibel 2011, Rimmer et al. 2012). CR ionization rates have also been measured in dense molecular clouds and massive stellar envelopes, by modeling the observed abundance of molecular ions like $\mathrm{HCO}^{+}$and $\mathrm{DCO}^{+}$. The values of $\zeta$ in these environments show considerable scatter, either intrinsic or due to uncertainties in the chemical modeling of the primary data. In any case the average value of $\zeta$ in dense molecular clouds is between one or two orders of magnitude smaller than in diffuse clouds. Fig. 1 shows a summary of the observational determination of $\zeta$ in clouds of column density $N\left(\mathrm{H}_{2}\right)$ ranging from $10^{20} \mathrm{~cm}^{-2}$ to $10^{24} \mathrm{~cm}^{-2}$. In addition to a considerable scatter, the data suggest a general trend of $\zeta$ decreasing with increasing $N\left(\mathrm{H}_{2}\right)$, as expected in simple 1-D models of CR attenuation (Padovani et al. 2009). Understanding the dependence of $\zeta$ on the physical characteristics of the environment (density, column density, magnetic fields, etc.) is a fundamental step for modeling the ionization fraction in the interstellar medium and the coupling of the gas with the magnetic field. This, in turn, has important consequences on theoretical models of cloud collapse and disk formation (Padovani et al. 2103, 2014).

\section{Cosmic-ray heating}

In addition to ionization, another process relating to the interaction of CRs with the interstellar medium is their role in heating the atomic and the molecular gas. CRs are an efficient (often dominant) source of heating in various environments, from the dense gas in molecular clouds (Goldsmith \& Langer 1978), both in normal and starbust galaxies (Suchkov et al. 1993), to photodissociation regions (Shaw et al. 2009), and possibly even in the primordial gas (Jasche et al. 2007).

Considering only molecular clouds in our Galaxy, the available observational data are consistent with a picture in which the dust temperature of prestellar cores (i.e. cores without any internal stellar energy source) in general decreases toward the center (Ward-Thompson et al. 2002, Bianchi et al. 2003). Observations in the mid-IR (Bacmann et al. 2000) and in the far-IR (Ward-Thompson et al. 2002) suggest dust temperature gradients consistent with heating from the external interstellar radiation field. Recent results obtained by the sub-mm satellite Herschel (e.g. Palmeirim et al. 2013) have confirmed these trends with a high degree of accuracy. On the theoretical side, available radiative transfer models predict a factor of $\sim 2$ increase in dust temperature from center to edge (Zucconi et al. 2001, Evans et al. 2001, Stematellos \& Whitworth 2003), with the gradient dependent on the cloud structure. 


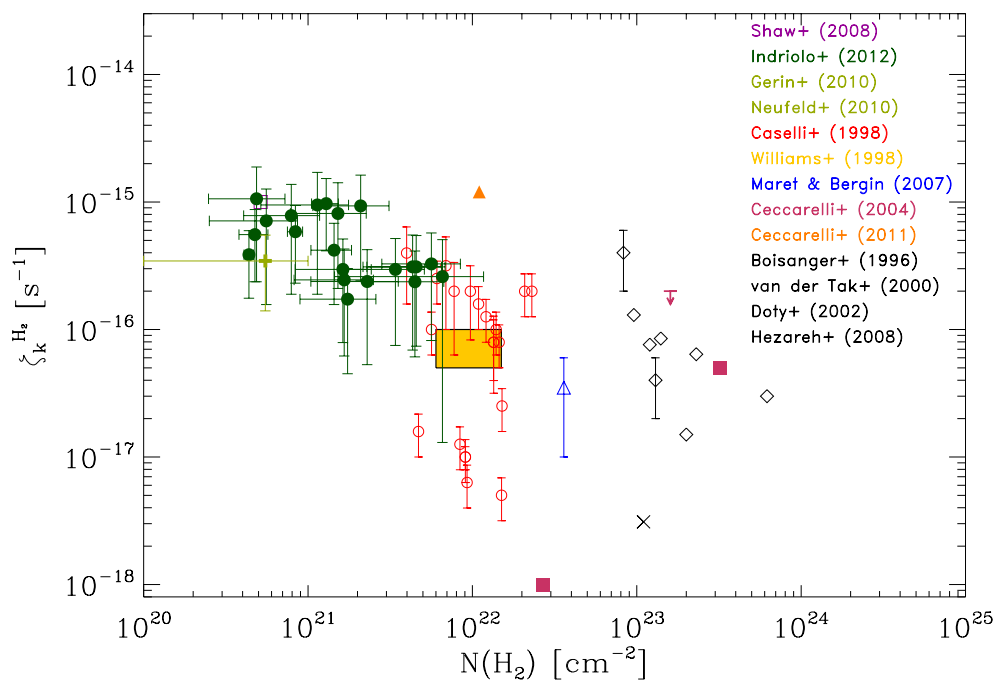

Figure 1: Values of the $\mathrm{CR}$ ionization rate of $\mathrm{H}_{2}$ in diffuse clouds (green dots), molecular cloud cores (red circles) and massive protostellar envelopes (black diamonds) as function of the cloud's column density. For references, see Padovani et al. (2009).

Additional observational constraints on the thermal structure of prestellar cores are given by spatially resolved measurements of the gas temperature that has been accurately traced by interferometric observations of molecular emission (in particular of $\mathrm{NH}_{3}$ ) in prestellar cores (Crapsi et al. 2007, Pagani et al. 2007) and dark globules (Pineda \& Bensch 2007). Low-mass cores show fairly uniform gas temperature (e.g. Tafalla et al. 2002 for L1517B and L1498), whereas massive quiescent cores in Orion show significant temperature drops from edge to center (Li et al. 2003), as predicted by theoretical models of dense, UV-shielded interstellar clouds (Falgarone \& Puget 1985, Galli et al. 2002). Thus, measurements of the gas temperature in prestellar cores can be used to constrain the $\mathrm{CR}$ ionization rate if radiative transfer models are also able to predict the gas temperature distribution resulting from the balance of the relevant heating and cooling mechanisms.

In general, the dust and gas temperatures $T_{\mathrm{d}}$ and $T_{\mathrm{g}}$ in a molecular cloud can be computed by solving simultaneously the equations of thermal balance of the gas and the dust

$$
\Gamma_{\text {ext }}=\Lambda_{\mathrm{d}}\left(T_{\mathrm{d}}\right)-\Lambda_{\mathrm{gd}}\left(T_{\mathrm{d}}, T_{\mathrm{g}}\right)
$$

and

$$
\Gamma_{\mathrm{CR}}=\Lambda_{\mathrm{g}}\left(T_{\mathrm{g}}\right)+\Lambda_{\mathrm{gd}}\left(T_{\mathrm{d}}, T_{\mathrm{g}}\right)
$$

where $\Gamma_{\text {ext }}$ is the dust heating rate per unit volume from the external radiation field, $\Lambda_{d}$ is the dust cooling rate by infrared emission, $\Gamma_{\mathrm{CR}}$ is the CR heating rate of the gas, $\Lambda_{\mathrm{g}}$ the gas cooling rate by molecular and atomic transitions, and $\Lambda_{\mathrm{gd}}$ the gas-dust energy transfer rate. Notice that we have ignored processes such as the photoelectric heating since we are interested in regions shielded by the external UV radiation field. For a detailed discussion of the heating and cooling functions that enter in the thermal balance equations see e.g. Goldsmith (2001) and Galli et al. (2002).

The CR heating $\Gamma_{\mathrm{CR}}$ is usually parametrized as

$$
\Gamma_{\mathrm{CR}}=n\left(\mathrm{H}_{2}\right) \zeta Q
$$




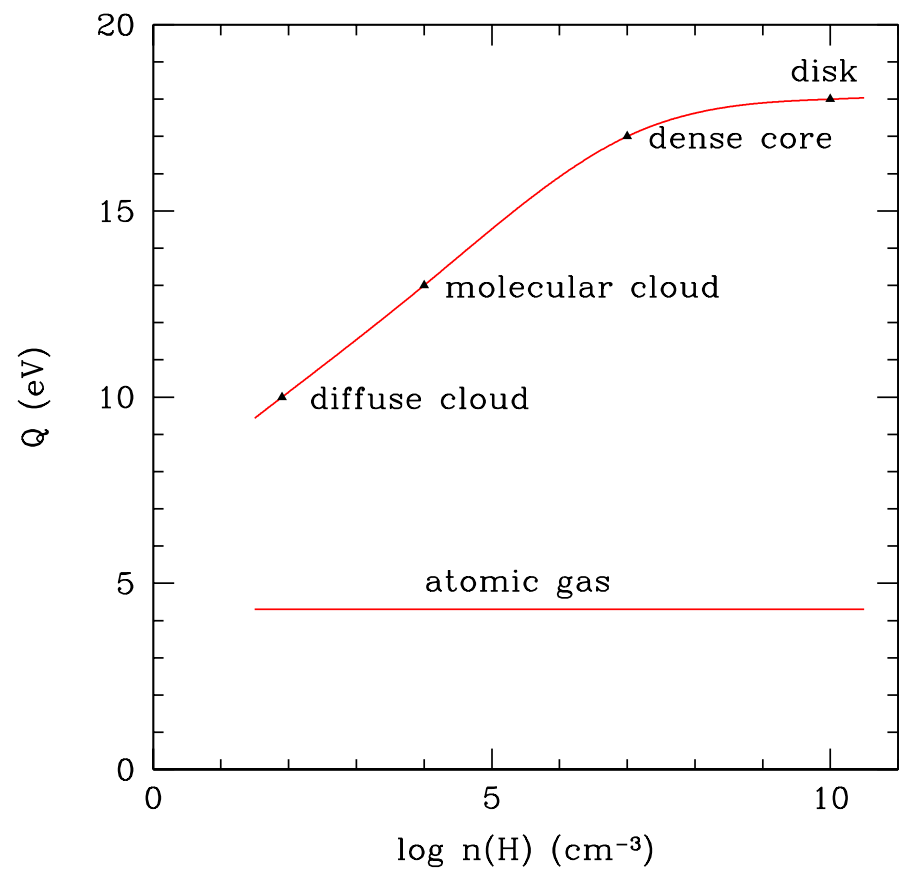

Figure 2: Average heat input per ionization $Q$ as function of the density of the environment for various astrophysical conditions (from Glassgold et al. 2012). Here $n(\mathrm{H})$ the density of hydrogen in all forms.

where $n\left(\mathrm{H}_{2}\right)$ is the $\mathrm{H}_{2}$ density and $Q$ is the mean heat input per ionization. Estimates of $Q$ available in the literature range over a factor of three (Glassgold \& Langer 1973a,b, Cravens et al. 1975, Cravens \& Dalgarno 1978, Goldsmith \& Langer 1978, Goldsmith 2001). Early studies suffered from the poorly known electron cross sections in the early 70s, the crude estimates of the energyloss functions, and ignored the roles of $\mathrm{H}^{+}$and $\mathrm{He}^{+}$ions in molecular gas were ignored. A more up to date and complete analysis was carried out by Dalgarno et al. (1999) who considered carefully all of the energy loss channels for electron energies up to $1 \mathrm{keV}$ in various mixtures of $\mathrm{H}_{1} \mathrm{H}_{2}$ and $\mathrm{He}$. They showed how the energy expended to make an ion pair is partitioned among elastic and several non-elastic processes, but they did not fully treat the heating. This was accomplished by Glassgold et al. (2012), who used the results of Dalgarno et al. (1999) to compute $Q$ with an accuracy of $\sim 20 \%$ in a mixture of $\mathrm{H}_{2}$ (or $\mathrm{H}$ ) and $\mathrm{He}$ for various astrophysical conditions (diffuse clouds, molecular clouds, dense molecular cloud cores and protostellar disks). As shown by Glassgold et al. (2012), in dense molecular regions about 50\% of the energy of the ejected electron can go into heating. In addition, CRs also produce ions and excited molecules that can interact with the dominant neutral atomic or molecular gas. The products of these reactions deposit in the gas a significant amount of the available energy in the form of chemical heating, that represents a significant part of the $\mathrm{CR}$ heating. The average heat input per ionization (including the chemical heating), computed by Glassgold et al. (2012) is shown schematically in Fig. [

Notice that a similar process arises in the X-ray irradiation of molecular regions. In fact, X-ray and CR ionization are closely related because the energy of the photon is almost entirely converted into energy of the primary photoelectron, and therefore the interactions of photons or CR electrons and nuclei with dense gas are largely determined by the many fast supra-thermal electrons they 
produce. Of course there are differences: X-ray ionization is accompanied by fast Auger electrons as well as secondaries, and CRs have an electron as well a nuclear component.

\section{A specific example: L1544}

As a specific example, we compare the results of the modelling described in the previous section with the gas temperature profile inferred from $\mathrm{NH}_{3}$ observations of the starless core $\mathrm{L} 1544$, a well-studied low-mass core in Taurus, which presents a large central density $\left(n_{\mathrm{c}}\left(\mathrm{H}_{2}\right)>10^{6} \mathrm{~cm}^{-3}\right)$. Several observational characteristics make this core a good candidate for being on the point of becoming unstable (Crapsi et al. 2005) and collapse to form stars.

To model the thermal structure of L1544, we adopt the parameters derived by Galli et al. (2002) to match the thermal dust emission map obtained by Ward-Thompson et al. (1999). To compute the gas temperature, we notice that the energy deposited in the gas by CR ionization is negligible when compared to the energy absorbed by the dust, so that the energy transfer between gas and dust will not significantly affect the grain temperature. Therefore, it is only necessary to solve the equation of thermal equilibrium of the gas. According to these models, in the inner part of L1544 (densities above $10^{5} \mathrm{~cm}^{-3}$ ), the gas temperature is coupled to that of the dust and thus decreases gradually toward the core center, where $T_{\mathrm{g}} \approx T_{\mathrm{d}} \approx 6 \mathrm{~K}$. For $\zeta>10^{-18} \mathrm{~s}^{-1}$ the gas is slightly hotter than the dust due to $\mathrm{CR}$ heating and this difference increases as the density decreases. In outer regions where the density is below $\sim 10^{5} \mathrm{~cm}^{-3}$ and where shielding from the external radiation field is much less, the gas and dust temperatures become uncoupled and one may find gas temperatures higher or lower than that of the dust, depending on the value of the CR ionization rate.

Fig. B shows the dust and gas temperature of our model, whereas the black and red points are the gas temperature determined from $\mathrm{NH}_{3}$ by Tafalla et al. (2002) with the Effelsberg single-dish radiotelescope and by Crapsi et al. (2007) with the VLA interferometer array, respectively. Since the models are spherically symmetric, the model temperature profiles are shown as a function of radius, whereas the data are plotted as a function of the projected distance from the dust peak. The interferometer data constrain the gas temperature in the inner regions of the core, showing a clear evidence of a temperature gradient from $\sim 6 \mathrm{~K}$ at the core's center to $\sim 8 \mathrm{~K}$ at a distance of $\sim 5000$ AU from the dust peak. The observed gradient is in agreement with theoretical predictions if the CR ionization rate is $\zeta \approx 10^{-17} \mathrm{~s}^{-1}$ (or lower). Notice that a value of $\zeta=10^{-16} \mathrm{~s}^{-1}$, typical of diffuse molecular clouds, results in a significantly higher gas temperature profile, and is inconsistent with the VLA data. These results compare favourably with the analysis of the chemical abundances of L1544: a best-fit chemical model reproducing the observed molecular column densities at the dust peak and the observed abundance profiles gives $\zeta=1.3 \times 10^{-17} \mathrm{~s}^{-1}$ (Vastel et al. 2006).

\section{Discussion and conclusions}

The example illustrated in the previous section shows how measurements of gas temperature in molecular cloud cores can be used to infer or constrain the CR ionization rate. This method may represent an alternative, or a complement, to the traditional approach based on the determination of abundance ratios of molecular ions (Guelin et al. 1977, Caselli 1998), that suffers from limitations 


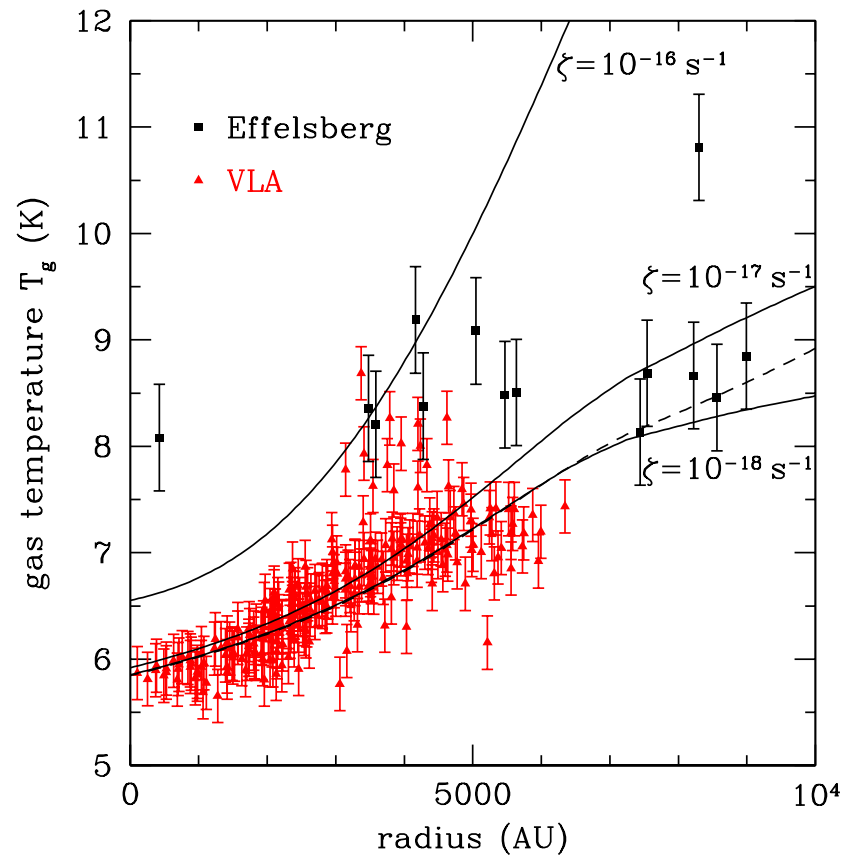

Figure 3: Thermal structure of the prestellar core L1544. The curves show the radial profile of the gas and dust temperature computed with the model of Galli et al. (2002) for different values of the CR ionization rate. From bottom to top: $\zeta=10^{-18} \mathrm{~s}^{-1}, 10^{-17} \mathrm{~s}^{-1}$, and $10^{-16} \mathrm{~s}^{-1}$ (solid curves, gas temperature; dashed curve, dust temperature). The data show the gas temperature derived from $\mathrm{NH}_{3}$ observations by Tafalla et al. (2002) and Crapsi et al. (2007) (black and red points, respectively).

associated to uncertainties in the chemical networks for dense clouds. Of course, as shown by the case of L1544, high-quality and high-resolution data are needed to determine with sufficient accuracy the gas temperature profile in molecular cloud cores (compare the Effelsberg and VLA data in Fig. (B]). In addition, the method is applicable to clouds (or cloud regions) of intermediate density: if the density is too low (say for clouds of visual extinction $A_{V}$ less than about 4), the heating of the gas is dominated by the photoelectric effect on dust grains (see e.g. Le Bourlot et al. 1993), whereas for densities larger than $\sim 10^{6}$ the gas is thermally coupled to the dust. In either case, in these regimes the gas temperature becomes insensitive to the local CR ionization rate.

The possibility of using measurements of the gas temperature to infer the CR ionization rate has already been tested on molecular clouds of external galaxies. In fact, heating by CRs has been invoked to explain the presence of hot $\mathrm{H}_{2}$ in the Galactic center and in starburst galaxies (Güsten et al. 1985, Lo et al. 1987). In particular, the temperature of molecular clouds in the starburst galaxy M82, $T_{\mathrm{g}} \approx 50-150 \mathrm{~K}$, is compatible with a CR ionization rate $\zeta \approx 4 \times 10^{-15} \mathrm{~s}^{-1}$ (Suchkov et al. 1993), much in excess of the Galactic values discussed in Section 1. This CR enhancement, resulting from the boosted supernova rate typical of a starburst galaxy, in turn implies a synchrotron emission in M82 about 500 times larger than in the disk of the Milky Way, in agreement with radio observations (Seaquist et al. 1985) and recent measurements of high-energy gamma-rays in M82 (VERITAS Collaboration et al. 2009). The idea here is that the nuclear component of $\mathrm{CRs}$ is responsible for heating the molecular gas and producing the gamma-ray emission, while 
the associated low-energy electronic component (primary and/or secondary) is responsible for the synchrotron radio emission.

\section{Acknowledgments}

DG and MP acknowledge the support of the CNRS-INAF PICS project "Pulsar wind nebulae, supernova remnants and the origin of cosmic rays". MP also acknowledges the support of the OCEVU Labex (ANR-11-LABX-0060) and the A*MIDEX project (ANR-11-IDEX-0001-02) funded by the "Investissements d'Avenir" French government programme managed by the ANR.

\section{References}

[1] Bacmann, A., André, P., Puget, J.-L., et al. 2000, A\&A, 361, 555

[2] Bianchi, S., Gonçalves, J., Albrecht, M., et al. 2003, A\&A, 399, L43

[3] Caselli, P., Walmsley, C. M., Terzieva, R., Herbst, E. 1998, ApJ, 499, 234

[4] Crapsi, A., Caselli, P., Walmsley, C. M., et al. 2005, ApJ, 619, 379

[5] Crapsi, A., Caselli, P., Walmsley, M. C., Tafalla, M. 2007, A\&A, 470, 221

[6] Cravens, T. E., Victor, G. A., Dalgarno, A. 1975, Plan. Space Sci., 23, 1059

[7] Cravens, T. E., Dalgarno, A. 1978, ApJ, 219, 750

[8] Dalgarno, A., Yan, M., Liu, W. 1999, ApJS, 125, 237

[9] Evans, N. J., II, Rawlings, J. M. C., Shirley, Y. L., Mundy, L. G. 2001, ApJ, 557, 193

[10] Everett, J. E., Zweibel, E. G. 2011, ApJ, 739, 60

[11] Falgarone, E., Puget, J. L. 1985, A\&A, 142, 157

[12] Galli, D., Walmsley, M., Gonçalves, J. 2002, A\&A, 394, 275

[13] Glassgold, A. E., Langer, W. D. 1973a, ApJ, 179, L147

[14] Glassgold, A. E., Langer, W. D. 1973b, ApJ, 186, 859

[15] Glassgold, A. E., Galli, D., Padovani, M. 2012, ApJ, 756, 157

[16] Goldsmith, P. F. 2001, ApJ, 557, 736

[17] Goldsmith, P. F., \& Langer, W. D. 1978, ApJ, 222, 881

[18] Guelin, M., Langer, W. D., Snell, R. L., Wootten, H. A. 1977, ApJL, 217, L165

[19] Güsten, R., Walmsley, C. M., Ungerechts, H., Churchwell, E. 1985, A\&A, 142, 381

[20] Hayakawa, S., Nishimura, S., Takayanagi, T. 1961, PASJ, 13, 184

[21] Indriolo, N., Geballe, T. R., Oka, T., McCall, B. J. 2007, ApJ, 671, 1736

[22] Indriolo, N., Fields, B. D., McCall, B. J. 2009, ApJ, 694, 257

[23] Indriolo, N., McCall, B. J. 2012, ApJ, 745, 91

[24] Jasche, J., Ciardi, B., Enßlin, T. A. 2007, MNRAS, 380, 417

[25] Le Bourlot, J., Pineau Des Forets, G., Roueff, E., \& Flower, D. R. 1993, A\&A, 267, 233 
[26] Li, D., Goldsmith, P. F., Menten, K. 2003, ApJ, 587, 262

[27] Lo, K. Y., Cheung, K. W., Masson, C. R., et al. 1987, ApJ, 312, 574

[28] Padovani, M., Galli, D., Glassgold, A. E. 2009, A\&A, 501, 619

[29] Padovani, M., Galli, D. 2011, A\&A, 530, A109

[30] Padovani, M., Hennebelle, P., Galli, D. 2013, A\&A, 560, A114

[31] Padovani, M., Galli, D., Hennebelle, P., et al. 2014, A\&A, 571, A33

[32] Pagani, L., Bacmann, A., Cabrit, S., Vastel, C. 2007, A\&A, 467, 179

[33] Palmeirim, P., André, P., Kirk, J., et al. 2013, A\&A, 550, A38

[34] Pineda, J. L., Bensch, F. 2007, A\&A, 470, 615

[35] Rimmer, P. B., Herbst, E., Morata, O., Roueff, E. 2012, A\&A, 537, A7

[36] Seaquist, E. R., Bell, M. B., Bignell, R. C. 1985, ApJ, 294, 546

[37] Shaw, G., Ferland, G. J., Henney, W. J., et al. 2009, ApJ, 701, 677

[38] Stamatellos, D., Whitworth, A. P. 2003, A\&A, 407, 941

[39] Suchkov, A., Allen, R. J., Heckman, T. M. 1993, ApJj, 413, 542

[40] Spitzer, L., Tomasko, M. G. 1968, ApJ, 152, 971

[41] Tafalla, M., Myers, P. C., Caselli, P., Walmsley, C. M., Comito, C. 2002, ApJ, 569, 815

[42] Vastel, C., Caselli, P., Ceccarelli, C., et al. 2006, ApJ, 645, 1198

[43] VERITAS Collaboration, Acciari, V. A., Aliu, E., et al. 2009, Nat., 462, 770

[44] Ward-Thompson, D., André, P., Kirk, J. M. 2002, MNRAS, 329, 257

[45] Zucconi, A., Walmsley, C. M., Galli, D. 2001, A\&A, 376, 650 